\title{
CONTINUITY AND CONVERGENCE PROPERTIES OF EXTREMAL-INTERPOLATING DISKS
}

\author{
PASCAL J. ThOMAS
}

\begin{abstract}
Let $a$ be a sequence of points in the unit ball of $\mathbb{C}^{n}$. Eric Amar and the author have introduced the nonnegative quantity $\rho(a)=$ $\inf _{\alpha} \inf _{k} \prod_{j: j \neq k} d_{G}\left(\alpha_{j}, \alpha_{k}\right)$, where $d_{G}$ is the Gleason distance in the unit disk and the first infimum is taken over all sequences $\alpha$ in the unit disk which map to $a$ by a map from the disk to the ball.

The value of $\rho(a)$ is related to whether $a$ is an interpolating sequence with respect to analytic disks passing through it, and if $a$ is an interpolating sequence in the ball, then $\rho(a)>0$.

In this work, we show that $\rho(a)$ can be obtained as the limit of the same quantity for the truncated finite sequences, and that $\rho(a)$ depends continuously on $a$ when $a$ is finite. Furthermore, we describe some of the behavior of the minimizing sequences of maps involved in the extremal problem used to define $\rho$.
\end{abstract}

\section{Introduction.}

This article is being written to provide some additional properties of the notion of extremal disks introduced in the joint paper $[\mathbf{A}-\mathbf{T}]$. It would not have arisen without the stimulating discussions I had with Eric Amar, for which I wish to thank him. Some of the genesis of this work took place while I was enjoying the hospitality of the University of California at Los Angeles and the University of Wisconsin-Madison, and my thanks go to them as well.

First we recap and simplify a few notations from $[\mathbf{A}-\mathbf{T}]$.

As usual, $\mathbb{D}$ denotes the unit disk in the complex plane, and $\mathbb{B}^{n}$ the unit ball in $\mathbb{C}^{n}: \mathbb{B}^{n}:=\left\{z \in \mathbb{C}^{n}\right.$ st $\left.z \cdot \bar{z}=\sum_{1}^{n}\left|z_{j}\right|^{2}<1\right\}$.

Let $a=\left\{a_{k}, k \in \mathbb{Z}_{+}^{*}\right\} \subset \mathbb{B}^{n}, \alpha=\left\{\alpha_{k}, k \in \mathbb{Z}_{+}^{*}\right\} \subset \mathbb{D}$, possibly finite sequences. Given $\phi$ a holomorphic map from $\mathbb{D}$ to $\mathbb{B}^{n}$, we shall employ the abbreviated notation $\phi(\alpha)=a$ to mean that for all $k \in \mathbb{Z}_{+}^{*}, \phi\left(\alpha_{k}\right)=a_{k}$. 
When $\alpha$ is such that a $\phi$ as above does exist, we say that $\alpha$ maps to $a$, and write $\alpha \mapsto a$ for short. It means that the map $\phi$ describes an analytic disk passing through the points of the sequence $a$ without "slopping over" the boundary of the ball.

Definition [A-T]. For $a \subset \mathbb{B}^{n}, n \geq 1, k \in \mathbb{Z}_{+}^{*}$,

$$
\rho_{k}(a):=\inf \left\{\delta_{k}(\alpha): \alpha \mapsto a\right\} ;
$$

and

$$
\rho(a):=\inf \{\delta(\alpha): \alpha \mapsto a\}
$$

where

$$
\begin{aligned}
\delta_{k}(\alpha) & :=\prod_{j: j \neq k} d_{G}^{\mathbb{D}}\left(\alpha_{k}, \alpha_{j}\right), \\
\delta(\alpha) & :=\inf _{k} \delta_{k}(\alpha),
\end{aligned}
$$

and, for $z$ and $w$ in a domain $\Omega, d_{G}^{\Omega}$ denotes the Gleason distance:

$$
d_{G}^{\Omega}(z, w):=\sup \left\{|f(w)|: f \in H^{\infty}(\Omega) \text { st } f(z)=0 \text { and }\|f\|_{\infty} \leq 1\right\} .
$$

Note that, for any nonnegative integer $n$,

$$
1-d_{G}^{\mathbb{B}^{n}}(z, w)^{2}=\frac{\left(1-|z|^{2}\right)\left(1-|w|^{2}\right)}{|1-z \cdot \bar{w}|^{2}},
$$

in particular

$$
d_{G}^{\mathbb{D}}(z, w)=\frac{|z-w|}{|1-z \bar{w}|}
$$

$($ see $[\mathbf{G a}],[\mathbf{R u}])$.

Definition $[\mathbf{A}-\mathbf{T}]$. We say that $\phi$ is an extremal-interpolating disk iff there exists an $\alpha \subset \mathbb{D}$ such that $\phi(\alpha)=a$ and $\delta(\alpha)=\rho(a)$.

Such disks are those for which the pre-image sequence $\alpha$ is, in a sense, as close together as it can within the unit disk (the Schwarz Lemma is preventing its points from being arbitrarily close to each other).

Results about existence of extremal-interpolating disks and some of their first properties were given in $[\mathbf{A}-\mathbf{T}]$ (where they were simply called "extremal disks"), as well as motivations for the study of this notion. Essentially, $\rho$ measures whether $a$ is an interpolating sequence with respect to holomorphic functions bounded on the analytic disks passing through it (as opposed as being interpolating with respect to functions bounded on the whole ball). 


\section{Convergence along finite subsequences and semi-continuity.}

\section{Theorem 1.}

$$
\begin{aligned}
& \text { Let } a=\left\{a_{k}, k \in \mathbb{Z}_{+}^{*}\right\} \subset \mathbb{B}^{n} \text {, then } \\
& \qquad \rho(a)=\lim _{N \rightarrow \infty} \rho\left(\left\{a_{k}, 1 \leq k \leq N\right\}\right)=\inf _{N \in \mathbb{Z}_{+}^{*}} \rho\left(\left\{a_{k}, 1 \leq k \leq N\right\}\right) .
\end{aligned}
$$

\section{Corollary 1.}

The function $a \mapsto \rho(a)$ is upper semi-continuous with respect to the topology given by the distance $d(a, b):=\sup _{k} d_{G}\left(a_{k}, b_{k}\right)$.

\section{Proof of Corollary 1:}

Lemma 2 in $[\mathbf{A}-\mathbf{T}]$ proved that the function $\rho$ is u.s.c. over the set of finite sequences with a given number of points, thus $a \mapsto \rho\left(\left\{a_{k}, 1 \leq k \leq\right.\right.$ $N\}$ ) is an u.s.c. function, and the greatest lower bound of a family of u.s.c. functions is also u.s.c.

\section{Corollary 2.}

Any sequence $a \subset \mathbb{B}^{n}$ verifying $\rho(a)>0$ is separated, i.e. there exists $\delta>0$ such that for any $j \neq k, d_{G}\left(a_{j}, a_{k}\right) \geq \delta$.

This Corollary is interesting in the context of interpolating sequences for bounded holomorphic functions (see $[\mathbf{C}],[\mathbf{G a}]$ for definitions). Separatedness is an easy necessary condition for a sequence of points to be an interpolating sequence. One also easily sees that $\rho(a)>0$ is another necessary condition for $a$ to be an interpolating sequence $[\mathbf{A}-\mathbf{T}]$. Thus we see that this new necessary condition implies a better-known one.

Proof of Corollary 2:

By renumbering the sequence, take $j=1, k=2$. It is easy to see (cf. $[\mathbf{A}-\mathbf{T}])$ that $\rho\left(\left\{a_{1}, a_{2}\right\}\right)=d_{G}\left(a_{1}, a_{2}\right)$. Apply Theorem 1 for $N=2$ : $d_{G}\left(a_{1}, a_{2}\right) \geq \rho(a)>0$.

Proof of Theorem 1:

Given any $\epsilon>0$, let $\alpha \subset \mathbb{D}$ be such that $\delta(\alpha)<\rho(a)+\epsilon$. Then there exists $k$ and $N$ such that

$$
\prod_{j: j \neq k, 1 \leq j \leq N} d_{G}\left(\alpha_{k}, \alpha_{j}\right)<\rho(a)+2 \epsilon .
$$

Thus by definition $\rho\left(\left\{a_{k}, 1 \leq k \leq N\right\}\right) \leq \rho(a)+2 \epsilon$, and we have

$$
\rho(a) \geq \inf _{N \in \mathbb{Z}_{+}^{*}} \rho\left(\left\{a_{k}, 1 \leq k \leq N\right\}\right) .
$$

The rest of the Theorem will follow from the 


\section{Proposition 3.}

Suppose $a$ is a sequence in $\mathbb{B}^{n}$ and $\phi$ a holomorphic map from $\mathbb{D}$ to $\mathbb{B}^{n}$, and, for $1 \leq k \leq N, \alpha_{k} \in \mathbb{D}$ such that $\phi\left(\alpha_{k}\right)=a_{k}$. Then, given any $\epsilon>0$, there exists a holomorphic map $\psi$ from $\mathbb{D}$ to $\mathbb{B}^{n}$ and a sequence $\beta \subset \mathbb{D}$ such that

$$
\psi(\beta)=a \text { and } \delta(\beta) \leq \delta\left(\left\{\alpha_{k}, 1 \leq k \leq N\right\}\right)+\epsilon .
$$

In particular, for any $N<M, \rho\left(\left\{a_{k}, 1 \leq k \leq N\right\}\right) \geq \rho\left(\left\{a_{k}, 1 \leq k \leq\right.\right.$ $M\})$.

The proof of Proposition 3 will be given in Section 4 .

End of Proof of Theorem 1:

The last clause of the proposition shows that the limit in the theorem exists and equals the infimum. Furthermore, by choosing a sequence $\alpha$ such that $\delta(\alpha) \leq \rho\left(\left\{a_{k}, 1 \leq k \leq N\right\}\right)+\epsilon$, we have

$$
\rho(a) \leq \delta(\beta) \leq \rho\left(\left\{a_{k}, 1 \leq k \leq N\right\}\right)+2 \epsilon,
$$

which proves the required inequality.

\section{Convergence of mappings.}

The first section lends some validation to our approach (in $[\mathbf{A}-\mathbf{T}]$ ) of studying the behavior of $\rho(a)$ mostly when $a$ is a finite sequence. In that case, since $\rho$ is defined as an infimum, it is legitimate to wonder what happens when we take a sequence of mappings $\phi^{p}$ and sequences $\alpha^{p}$ such that, for any $p, \phi_{p}\left(\alpha^{p}\right)=a$, and $\lim _{p \rightarrow \infty} \delta\left(\alpha^{p}\right)=\rho(a)$. By Montel's theorem, a subsequence of $\left\{\phi^{p}\right\}_{p}$ will converge uniformly on compact subsets of $\mathbb{D}$, but no such convergence is guaranteed for the points in the sequences $\alpha^{p}$, so the question arises of what the relationship between the limit of the mappings and the original sequence.

\section{Normalizations.}

Since we are dealing with a finite sequence $a$, we may always assume (after re-numbering) that $\rho(a)=\rho_{1}(a)$, and shall do so for the remainder of this section.

Likewise, when have a sequence $\alpha$ which maps to $a$, by applying an automorphism of the disk, we reduce ourselves to the case where $\alpha_{1}=0$.

We introduce a class of special holomorphic mappings from the disk to the ball: 


\section{Definition.}

We say that $\phi$, a holomorphic map from $\mathbb{D}$ to $\mathbb{B}^{n}$, is a ball-valued Blaschke product of degree $N$ iff for $\zeta \in \mathbb{D}$,

$$
\phi(\zeta)=\left(\frac{P_{1}(\zeta)}{Q(\zeta)}, \ldots, \frac{P_{n}(\zeta)}{Q(\zeta)}\right)
$$

where $P_{1}, \ldots, P_{n}$ and $Q$ are polynomials with $\max \left(\operatorname{deg} P_{j}, 1 \leq j \leq\right.$ $n, \operatorname{deg} Q)=N, Q$ has no zeros in $\overline{\mathbb{D}}$ and for any $\zeta$ such that $|\zeta|=1$,

$$
1=|\phi(\zeta)|^{2}=\sum_{1}^{n} \frac{\left|P_{j}(\zeta)\right|^{2}}{|Q(\zeta)|^{2}}
$$

The following was essentially proved in $[\mathbf{A}-\mathbf{T}$, Theorem 1]:

\section{Theorem.}

If $a=\left\{a_{k}, 1 \leq k \leq N\right\} \subset \mathbb{B}^{n}, \alpha \subset \mathbb{D}$, with $\phi$ a holomorphic map from $\mathbb{D}$ to $\mathbb{B}^{n}$, such that $\phi(\alpha)=a$, and $\delta_{1}(\alpha)=\rho_{1}(a)$ (in particular if $\phi$ gives an extremal-interpolating disk for a) then $\phi$ is a ball-valued Blaschke product of degree no greater than $N-1$, uniquely determined by $\alpha$.

Only the precise form of the rational map was not explicitly given in $[\mathbf{A}-\mathbf{T}]$, but it is easy to obtain by following the induction performed there, observing that at each step we only perform composition by Möbius automorphisms of the disc or ball, and multiplication by $\zeta$.

We can now state:

\section{Theorem 2.}

Let $a=\left\{a_{k}, 1 \leq k \leq N\right\} \subset \mathbb{B}^{n}$, and $\left\{\phi^{p}\right\}_{p}$ a sequence of mappings from the disk to the ball such that for each $p, \phi^{p}\left(\alpha_{k}^{p}\right)=a_{k}, 1 \leq k \leq N$, where $\alpha_{k}^{p} \in \mathbb{D}, \alpha_{1}^{p}=0$, and

$$
\lim _{p \rightarrow \infty} \delta_{1}\left(\alpha^{p}\right)=\lim _{p \rightarrow \infty} \prod_{k=2}^{N}\left|\alpha_{k}^{p}\right|=\rho(a) .
$$

Then there exist subsequences, denoted again by $\left\{\phi^{p}\right\}_{p}$ and $\left\{\alpha^{p}\right\}_{p}$, a ball-valued Blaschke product $\phi$, and a sequence $\alpha=\left\{\alpha_{k}, 1 \leq k \leq N\right\} \subset$ $\overline{\mathbb{D}}$ such that

(i) $\lim _{p \rightarrow \infty} \phi^{p}=\phi$, uniformly on compact sets of $\mathbb{D}$,

(ii) $\lim _{p \rightarrow \infty} \alpha_{k}^{p}=\alpha_{k} \in \overline{\mathbb{D}}$, and

(iii) $\phi\left(\alpha_{k}\right)=a_{k}$ iff $\left|\alpha_{k}\right|<1$. 
Let $S:=\left\{k \in\{1, \ldots, N\}\right.$ st $\left.\left|\alpha_{k}\right|<1\right\}$. Then $\rho\left(\left\{a_{k}, k \in S\right\}\right)=\rho(a)$ and $\phi$ is of degree no greater than $\# S-1$.

\section{Remarks.}

This theorem says that we do have convergence towards some extremal-interpolating disk, but passing only through a subsequence of $a$.

In the case where $N=3$ and $a$ itself does not admit an extremalinterpolating disk, we see that the theorem implies that a subsequence of the minimizing sequence of mappings has to converge to an affine disk through two of the points of the original sequence.

\section{Proof of Theorem 2:}

By Montel's Theorem and compactness of $\overline{\mathbb{D}}$, it is easy to extract subsequences having properties (i) and (ii). The "if" part of (iii) follows by equicontinuity of the converging subsequence of maps.

Now

$$
\begin{aligned}
\rho(a) & =\rho_{1}(a)=\lim _{p \rightarrow \infty} \delta_{1}\left(\alpha^{p}\right)=\lim _{p \rightarrow \infty}\left(\prod_{k \geq 2, k \in S}\left|\alpha_{k}^{p}\right| \prod_{k \notin S}\left|\alpha_{k}^{p}\right|\right) \\
& =\delta_{1}\left(\left\{\alpha_{k}, k \in S\right\}\right) \geq \rho_{1}\left(\left\{a_{k}, k \in S\right\}\right) \geq \rho\left(\left\{a_{k}, k \in S\right\}\right),
\end{aligned}
$$

which itself is no less than $\rho(a)$ by Proposition 3, so we actually have equality throughout. Since $\phi\left(\left\{\alpha_{k}, k \in S\right\}\right)=\left\{a_{k}, k \in S\right\}$, and $\delta_{1}\left(\left\{\alpha_{k}, k \in S\right\}\right)=\rho_{1}\left(\left\{a_{k}, k \in S\right\}\right), \phi$ is a ball-valued Blaschke product of degree no greater than $\# S-1$, so that for $k \notin S,\left|\phi\left(\alpha_{k}\right)\right|=1$, so that $\phi\left(\alpha_{k}\right) \neq a_{k}$, which finally proves the "only if" part of (iii).

It would be nicer to be able to describe the subsequence through which an extremal-interpolating disk passes, $\left\{a_{k}, k \in S\right\}$, in terms of the sequence $a$. Observe that $\left\{a_{k}, k \in S\right\}$ is a sequence with the same $\rho$ as the original sequence. If such a subset $S$ is given, we have the:

\section{Theorem 3.}

Let $S \subset\{1, \ldots, N\}$, such that $1 \in S$, be minimal for the property that

$$
\rho_{1}\left(\left\{a_{k}, k \in S\right\}\right)=\rho_{1}(a)=\rho(a) .
$$

Then there exists a sequence of finite sequences in the disk, $\left\{\alpha^{p}\right\}_{p}$ and a sequence of mappings from the disk to the ball $\left\{\phi^{p}\right\}_{p}$ such that

(i) $\phi^{p}\left(\alpha^{p}\right)=a$,

(ii) $\rho_{1}(a)=\lim _{p \rightarrow \infty} \delta_{1}\left(\alpha^{p}\right)$, 
(iii) $\lim _{p \rightarrow \infty} \alpha_{k}^{p}=\alpha_{k} \in \overline{\mathbb{D}}$ and $S=\left\{k \in\{1, \ldots, N\}\right.$ st $\left.\left|\alpha_{k}\right|<1\right\}$,

(iv) $\lim _{p \rightarrow \infty} \phi^{p}=\phi$, a ball-valued Blaschke product of degree no greater than $\# S-1$, and

(v) $\phi\left(\left\{\alpha_{k}, k \in S\right\}\right)=\left\{a_{k}, k \in S\right\}, \delta_{1}\left(\left\{\alpha_{k}, k \in S\right\}\right)=\rho_{1}\left(\left\{a_{k}, k \in\right.\right.$ $S\})=\rho_{1}(a)=\rho(a)$.

\section{Proof of Theorem 3:}

The sufficient condition for $\rho$ to be attained given in [A-T, Lemma 3] covered the special case where the only such set $S$ is the whole $\{1, \ldots, N\}$. The present proof will use the same idea.

For a given $p$, pick first, using the definition of $\rho_{1}$, a sequence $\left\{\beta_{k}^{p}, k \in\right.$ $S$ \} so that

$$
\delta_{1}\left(\left\{\beta_{k}^{p}, k \in S\right\}\right) \leq \rho_{1}\left(\left\{a_{k}, k \in S\right\}\right)+\frac{1}{p} ;
$$

then modify and complete this sequence according to Proposition 3 to get $\alpha^{p}=\left\{\alpha_{k}^{p}, 1 \leq k \leq N\right\}$ so that

$$
\rho_{1}(a) \leq \delta_{1}\left(\left\{\alpha^{p}\right\}\right) \leq \rho_{1}\left(\left\{a_{k}, k \in S\right\}\right)+\frac{2}{p}=\rho_{1}(a)+\frac{2}{p} .
$$

This forces $\lim _{p \rightarrow \infty}\left|\alpha_{k}^{p}\right|=1$ for $k \notin S$.

On the other hand, for $k \in S$, by the minimality property of $S$, $\rho_{1}\left(\left\{a_{j}, j \in S \backslash\{k\}\right\}\right)>\rho(a)$. Then

$$
\left|\alpha_{k}^{p}\right|=\frac{\prod_{j \in S}\left|\alpha_{j}^{p}\right|}{\prod_{j \in S, j \neq k}\left|\alpha_{j}^{p}\right|} \leq \frac{\prod_{j \in S}\left|\alpha_{j}^{p}\right|}{\rho_{1}\left(\left\{a_{j}, j \in S \backslash\{k\}\right\}\right)} \leq \gamma_{k}<1,
$$

for $p$ large enough. Taking subsequences as before, we get the convergence of the points $\alpha_{k}^{p}$ to limits within the open disk when $k \in S$, and of the mappings to a mapping $\phi$. We obtain (v) as in the previous proof, and the resulting extremality forces to $\phi$ to be a ball-valued Blaschke product.

\section{Questions.}

Is the set $S$ of Theorem 2 always "minimal", i.e. of the type given in Theorem 3? It is clear that it contains a "minimal" set $S^{\prime}$, and that any "minimal" set that contains it must be equal to it. Also, the set $S$ is included in some set $S^{\prime \prime}$ maximal for the property that an extremalinterpolating disk does pass through $\left\{a_{k}, k \in S^{\prime \prime}\right\}$; must $S, S^{\prime}$ and $S^{\prime \prime}$ coincide?

More modestly, are there examples of sequences where several different minimal sets $S^{\prime}$ can be found? 


\section{Continuity in the finite case.}

Despite their limitations, the ideas of the previous section enable us to prove the following continuity result:

\section{Theorem 4.}

For every $N \in \mathbb{Z}_{+}^{*}$, the function $\left\{a_{k}, 1 \leq k \leq N\right\} \mapsto \rho\left(\left\{a_{k}, 1 \leq k \leq\right.\right.$ $N\})$ is continuous from $\left(\mathbb{B}^{n}\right)^{N}$ to $\mathbb{R}_{+}$.

Proof of Theorem 4:

We shall adopt the same normalisations as those in the previous section.

Since $\rho(a)=\min _{1 \leq k \leq N} \rho_{k}(a)$, it will be enough to prove that $\rho_{1}$ is a continuous function. By Lemma 2 in $[\mathbf{A}-\mathbf{T}]$, we already know it to be upper semi-continuous. We shall proceed by induction on $N$. Since $\rho\left(\left\{a_{1}, a_{2}\right\}\right)=d_{G}\left(a_{1}, a_{2}\right)$, the case $N=2$ is clear.

Now suppose the property true for all sequences $a$ such that $\# a \leq$ $N-1$. Given $a \in\left(\mathbb{B}^{n}\right)^{N}$, assume, to get a contradiction, that there is a sequence of sequences $a^{p} \subset\left(\mathbb{B}^{n}\right)^{N}$ such that $\lim _{p \rightarrow \infty} \rho_{1}\left(a^{p}\right)<\rho_{1}(a)$. Then for any proper subset $S \subset\{1, \ldots, N\}$, by the induction hypothesis,

$$
\lim _{p \rightarrow \infty} \rho_{1}\left(\left\{a_{k}^{p}, k \in S\right\}\right)=\rho_{1}\left(\left\{a_{k}, k \in S\right\}\right) \geq \rho_{1}(a),
$$

by Proposition 3. Therefore for $p$ large enough,

$$
\rho_{1}\left(a^{p}\right)<\min \left\{\rho_{1}\left(\left\{a_{k}^{p}, k \in S\right\}\right): S \subset\{1, \ldots, N\}, \# S \leq N-1\right\},
$$

so an application of $[\mathbf{A}-\mathbf{T}$, Lemma 3] shows that for each such $p$ there exists a sequence $\alpha^{p}$ in the disk such that $\delta_{1}\left(\alpha^{p}\right)=\rho_{1}\left(a^{p}\right)$ and a mapping $\phi^{p}$ such that $\phi^{p}\left(\alpha^{p}\right)=a^{p}$.

Now, for $\epsilon$ small enough, $p$ large enough, and any $k \in\{2, \ldots, N\}$, we have

$$
\left|\alpha_{k}^{p}\right|=\frac{\delta_{1}\left(\alpha^{p}\right)}{\delta_{1}\left(\left\{\alpha_{j}^{p}, j \neq k\right\}\right)} \leq \frac{\rho_{1}\left(a^{p}\right)}{\rho_{1}\left(\left\{a_{j}^{p}, j \neq k\right\}\right)} \leq \frac{\lim _{p \rightarrow \infty} \rho_{1}\left(a^{p}\right)}{\rho_{1}(a)}+\epsilon<1 .
$$

This implies that all the points $\alpha_{k}^{p}$ remain in a relatively compact disk within the unit disk, therefore by extracting a subsequence we may assume that for each $k, \lim _{p \rightarrow \infty} \alpha_{k}^{p}=\alpha_{k} \in \mathbb{D}$, and $\lim _{p \rightarrow \infty} \phi^{p}=\phi$, with uniform convergence on compact subsets of the unit disk. This implies that $\phi(\alpha)=a$, but since

$$
\delta_{1}(\alpha)=\lim _{p \rightarrow \infty} \delta_{1}\left(\alpha^{p}\right)=\lim _{p \rightarrow \infty} \rho_{1}\left(a^{p}\right)<\rho_{1}(a),
$$

we get a contradiction with the definition of $\rho_{1}$. 


\section{Proof of Proposition 3.}

As usual, we may assume without loss of generality that $\alpha_{1}=0$ and $\delta(\alpha)=\delta_{1}(\alpha)$, where $\alpha=\left\{\alpha_{j}\right\}_{1 \leq j \leq N}$. Also, renumber $a$ so that $\left|a_{j+1}\right| \geq$ $\left|a_{j}\right|$ for any $j \geq N+1$.

We write $M_{\beta}$ for the constant of interpolation of a sequence $\beta$, i.e.

$M_{\beta}:=\inf \left\{M>0\right.$ st $\forall a \subset \mathbb{B}^{n}$, there is $\phi: \mathbb{D} \rightarrow \mathbb{B}^{n}(0, M)$ st $\left.\phi(\beta)=a\right\}$.

Pick $r_{0}<1$ such that $\alpha \subset \mathbb{D}\left(0, r_{0}\right)$ and

$$
\prod_{j=2}^{N}\left|\frac{\alpha_{j}}{r_{0}}\right|<\delta_{1}(\alpha)+\epsilon
$$

and $\epsilon_{1}>0$ small enough so that $\epsilon_{1} \leq \frac{1-\left|a_{N+1}\right|}{M_{\alpha}+2}$ and $\phi\left(\overline{\mathbb{D}}\left(0, r_{0}\right)\right) \subset$ $\mathbb{B}^{n}\left(0,1-\left(M_{\alpha}+2\right) \epsilon_{1}\right)$.

Lemma 4. There exists a holomorphic function $E \in A(\overline{\mathbb{D}})$ (i.e. continuous up to the boundary) so that

(i) $|E(\zeta)| \leq 1$, for any $\zeta \in \overline{\mathbb{D}}$;

(ii) $|E(\zeta)-1| \leq \epsilon_{1}$, for any $\zeta \in \overline{\mathbb{D}}\left(0, r_{0}\right)$;

(iii) $E(1)=0$, but $E(\zeta) \neq 0$ for any $\zeta \in \mathbb{D}$.

Proof:

For $a \in(-1,+1)$ let $\phi_{a}(\zeta)=\frac{\zeta-a}{1-a \zeta}$, and $E(\zeta)=\left(1-\phi_{a}(\zeta)\right) / 2$. Then $E(1)=0$ and $\|E\|_{\infty} \leq 1$.

But the disk $\phi_{a}\left(\overline{\mathbb{D}}\left(0, r_{0}\right)\right)$ admits the line segment $\left[\frac{-r_{0}-a}{1+a r_{0}} ; \frac{r_{0}-a}{1-a r_{0}}\right]$ for its diameter and $\lim _{a \rightarrow 1^{-}} \frac{r_{0}-a}{1-a r_{0}}=-1$, so that for $a$ close enough to 1 , $\phi_{a}\left(\overline{\mathbb{D}}\left(0, r_{0}\right)\right) \subset D\left(-1,2 \epsilon_{1}\right)$, which yields (ii).

Lemma 5. There exists $F \in \mathcal{C}^{0}\left(\left[r_{0}, 1\right), \mathbb{B}^{n}\right)$ and $\left\{\alpha_{j}\right\}_{j \geq N+1}$ a strictly increasing sequence such that

(i) $F\left(\alpha_{j}\right)=a_{j}$ for $j \geq N+1$;

(ii) $M_{\left\{\alpha_{j}\right\}_{j \geq 1}} \leq M_{\left\{\alpha_{j}\right\}_{1 \leq j \leq N}}+1$;

(iii) $1-|F(x)| \geq \epsilon_{1}\left(M_{\alpha}+2\right)|E(x)|$, for any $x \in\left[r_{0}, 1\right)$;

(iv) $F\left(r_{0}\right)=\phi\left(r_{0}\right)$.

Proof: Once the $\alpha_{j}$ are given, we will define $F$ by linear interpolation:

$$
\begin{aligned}
F\left(\theta r_{0}+(1-\theta) \alpha_{N+1}\right) & :=\theta \phi\left(r_{0}\right)+(1-\theta) a_{N+1} \\
\text { and } F\left(\theta \alpha_{j}+(1-\theta) \alpha_{j+1}\right) & :=\theta a_{j}+(1-\theta) a_{j+1},
\end{aligned}
$$


so that

$$
\begin{aligned}
\min _{x \in\left[r_{0}, \alpha_{N+1}\right]}(1-|F(x)|) & =\min \left(1-\left|\phi\left(r_{0}\right)\right|, 1-\left|a_{N+1}\right|\right) \\
\min _{x \in\left[\alpha_{j}, \alpha_{j+1}\right]}(1-|F(x)|) & =\min \left(1-\left|F\left(\alpha_{j}\right)\right|, 1-\left|F\left(\alpha_{j+1}\right)\right|\right) \\
& =\min \left(1-\left|a_{j}\right|, 1-\left|a_{j+1}\right|\right)=1-\left|a_{j+1}\right| .
\end{aligned}
$$

By the choice of $\epsilon_{1}$,

$$
\left|E\left(r_{0}\right)\right| \leq 1 \leq \frac{1}{\left(M_{\alpha}+2\right) \epsilon_{1}} \min \left(1-\left|\phi\left(r_{0}\right)\right|, 1-\left|a_{N+1}\right|\right) .
$$

Choose $\alpha_{N+1}>r_{0}$ and inductively for $j \geq N+1, \alpha_{j+1}>\alpha_{j}$, large enough so that, for any $j \geq N$,

$$
\left|E\left(\alpha_{j+1}\right)\right| \leq \frac{1-\left|a_{j+2}\right|}{\left(M_{\alpha}+2\right) \epsilon_{1}}
$$

and

$$
M_{\left\{\alpha_{k}, 1 \leq k \leq j+1\right\}} \leq M_{\left\{\alpha_{k}, 1 \leq k \leq j\right\}}+2^{N-j-1} .
$$

The first condition can be met since $E(1)=0$. In order to estimate the constant $M_{\left\{\alpha_{k}, k \leq j+1\right\}}$, let $E_{1}$ be a function as in Lemma 4, this time with $r_{0}^{\prime}=\left|\alpha_{j}\right|, \epsilon_{1}^{\prime}=2^{N-j-2} M_{\left\{\alpha_{k}, k \leq j\right\}}$.

Suppose $\left\{v_{k}, k \leq j+1\right\}$ is a sequence of values in the unit ball. Pick a map $f_{1}$ such that $f_{1}\left(\alpha_{k}\right)=\frac{v_{k}}{E_{1}\left(\alpha_{k}\right)}$ for $k \leq j$, and $\left\|f_{1}\right\|_{\infty, n} \leq$ $\left(1-\epsilon_{1}^{\prime}\right)^{-1} M_{\left\{\alpha_{k}, k \leq j\right\}}$.

Choose $\rho>0$ small enough so that $\left|E_{1}(\zeta)\right| \leq \epsilon_{1}^{\prime} M_{\left\{\alpha_{k}, k \leq j\right\}}$ for $\zeta \in$ $D(1, \rho)$; pick $f_{2}(\zeta)=\left(\frac{1+\zeta}{2}\right)^{N}$, with $N$ large enough so that $\left|f_{2}(\zeta)-1\right| \leq$ $\epsilon_{1}^{\prime} M_{\left\{\alpha_{k}, k \leq j\right\}}$ for $\zeta \in \overline{\mathbb{D}} \backslash D(1, \rho)$. Now we take $\alpha_{j+1}>1-\rho$, and use as interpolating function

$$
f(\zeta):=E_{1}(\zeta) f_{1}(\zeta)+v_{j+1} \frac{f_{2}(\zeta)}{f_{2}\left(\alpha_{j+1}\right)} .
$$

Thus for $\zeta \in \overline{\mathbb{D}} \backslash D(1, \rho)$,

$$
\begin{aligned}
|f(\zeta)| & \leq\left(1-\epsilon_{1}^{\prime}\right)^{-1} M_{\left\{\alpha_{k}, k \leq j\right\}}+\left(1-\epsilon_{1}^{\prime}\right)^{-1} \epsilon_{1}^{\prime} M_{\left\{\alpha_{k}, k \leq j\right\}} \\
& \leq M_{\left\{\alpha_{k}, k \leq j\right\}}\left(1+3 \epsilon_{1}^{\prime}\right)<M_{\left\{\alpha_{k}, k \leq j\right\}}+2^{N-j},
\end{aligned}
$$

and a similar estimate is true for $\zeta \in D(1, \rho)$.

We shall need the following simple modification of Brelot's proof of Carleman's theorem, see $[\mathbf{G l}]$ and $[\mathbf{K a}]$. 


\section{Lemma 6.}

For any $G \in \mathcal{C}^{0}\left(\overline{\mathbb{D}}\left(0, r_{0}\right) \cup\left[r_{0}, 1\right) ; \mathbb{B}^{n}\right) \cap \mathcal{H}\left(\mathbb{D}\left(0, r_{0}\right)\right)$, for any $e \in$ $\mathcal{C}^{0}\left(\overline{\mathbb{D}}\left(0, r_{0}\right) \cup\left[r_{0}, 1\right) ; \mathbb{R}_{+}^{*}\right)$, there exists $f_{1} \in \mathcal{H}(\mathbb{D})$ such that

$$
\text { For any } \zeta \in \overline{\mathbb{D}}\left(0, r_{0}\right) \cup\left[r_{0}, 1\right), \quad\left|f_{1}(\zeta)-G(\zeta)\right|<e(\zeta) .
$$

Proof:

Choose a strictly increasing sequence $r_{0}<r_{1}<\cdots r_{m}<r_{m+1} \rightarrow 1$. Let $\left\{e_{m}\right\}_{m \geq 0}$ be a strictly decreasing sequence of positive numbers such that

$$
\begin{aligned}
e_{0} & <\min _{|\zeta| \leq r_{0} \text { or } r_{0} \leq \zeta \leq r_{1}}|e(\zeta)|, \\
e_{m} & <\min _{x \in\left[r_{m}, r_{m+1}\right]}|e(x)|, \quad m \geq 1,
\end{aligned}
$$

and $\lim _{m \rightarrow \infty} e_{m}=0$. Let $d_{m}:=e_{m}-e_{m+1}>0$ for $m \geq 0$.

We shall set $f_{1}=\lim _{m \rightarrow \infty} p_{m}$, where the $p_{m}$ are polynomial mappings which will be defined inductively as follows.

First let $g_{0}:=G$ on $\overline{\mathbb{D}}\left(0, r_{0}\right) \cup\left[r_{0}, r_{1}\right]$.

Now, for any $m \geq 1$, suppose we have already chosen continuous maps $g_{0}, \ldots, g_{m-1}$ and polynomial mappings $p_{1}, \ldots, p_{m-1}$ such that for any $k$ in the relevant ranges,

$$
\begin{aligned}
& g_{k} \in \mathcal{C}^{0}\left(\overline{\mathbb{D}}\left(0, r_{k}\right) \cup\left[r_{k}, r_{k+1}\right] ; \mathbb{B}^{n}\right), \\
& \left|g_{k}(x)-G(x)\right| \leq d_{k} \text { for any } x \in\left[r_{k}, r_{k+1}\right], \\
& g_{k}\left(r_{k+1}\right)=G\left(r_{k+1}\right)
\end{aligned}
$$

and

(iv) $\quad$ For any $\zeta \in \overline{\mathbb{D}}\left(0, r_{k-1}\right) \cup\left[r_{k-1}, r_{k}\right], \quad\left|p_{k}(\zeta)-g_{k-1}(\zeta)\right|<d_{k}$.

By Mergelyan's theorem (applied to each component function), we can choose $p_{m}$ a polynomial mapping such that

For any $\zeta \in \overline{\mathbb{D}}\left(0, r_{m-1}\right) \cup\left[r_{m-1}, r_{m}\right], \quad\left|p_{m}(\zeta)-g_{m-1}(\zeta)\right|<d_{m}$,

so it clearly verifies (iv) for $k=m$, and we define

$$
\begin{aligned}
g_{m}(\zeta) & :=p_{m}(\zeta) \text { for } \zeta \in \overline{\mathbb{D}}\left(0, r_{m}\right) \\
g_{m}(\zeta) & :=G(\zeta)+\left(p_{m}\left(r_{m}\right)-G\left(r_{m}\right)\right) \frac{\zeta-r_{m+1}}{r_{m}-r_{m+1}} \text { for } \zeta \in\left[r_{m}, r_{m+1}\right]
\end{aligned}
$$


To verify (i) for $k=m$, it's enough to notice that $g_{m}\left(r_{m}\right)=p_{m}\left(r_{m}\right)$; (iii) is clear; (ii) follows from the fact that $\left|p_{m}\left(r_{m}\right)-G\left(r_{m}\right)\right|=\mid p_{m}\left(r_{m}\right)-$ $g_{m-1}\left(r_{m}\right) \mid<d_{m}$, by (iii) for $k=m-1$ and the definition of $p_{m}$.

The sequence $\left\{p_{m}\right\}$ will converge uniformly on compact subsets of $\mathbb{D}$ since on any given $\overline{\mathbb{D}}\left(0, r_{m}\right)$, for any $k \geq m$,

$$
\sum_{j \geq k}\left|p_{j+1}-p_{j}\right|=\sum_{j \geq k}\left|p_{j+1}-g_{j}\right| \leq \sum_{j \geq k} d_{j+1}=e_{k+1} .
$$

Furthermore, on the interval $\left[r_{m}, r_{m+1}\right]$,

$$
\begin{aligned}
|p-G| & \leq\left|p-p_{m+1}\right|+\left|p_{m+1}-g_{m}\right|+\left|g_{m}-G\right| \\
& \leq \sum_{j \geq m+2} d_{j}+d_{m+1}+d_{m}=e_{m} \leq e
\end{aligned}
$$

For $m=0$, this also applies to points in the disc $\overline{\mathbb{D}}\left(0, r_{0}\right)$, so we're done.

Let $G$ be the map in $\mathcal{C}^{0}\left(\overline{\mathbb{D}}\left(0, r_{0}\right) \cup\left[r_{0}, 1\right), \mathbb{B}^{n}\right)$ obtained by "gluing together" the map $\phi$ given in the hypotheses of Proposition 3 and the function $F$ from Lemma 5. We take $e(\zeta)=\epsilon_{1}|E(\zeta)|$, and apply Lemma 6 to this $G$ to obtain a map $f_{1}$.

Recall the notation

$$
\|f\|_{n, \infty}=\sup \{|f(\zeta)|: \zeta \in \mathbb{D}\}
$$

where $f$ is a map from the disk to $\mathbb{C}^{n}$.

By our choices of constants of interpolation, the interpolation problem at the points $\alpha_{j}$ with data $\frac{-f_{1}\left(\alpha_{j}\right)+G\left(\alpha_{j}\right)}{E\left(\alpha_{j}\right)}$ can be solved by a holomorphic map $h$ with

$$
\|h\|_{n, \infty} \leq \epsilon_{1}\left(M_{\left\{\alpha_{j}\right\}_{j \geq 1}}\right) \leq \epsilon_{1}\left(M_{\alpha}+1\right) .
$$

Let $f_{2}=f_{1}+h E$; this is a holomorphic map such that $f_{2}(\alpha)=a$, and

$$
\left|f_{2}(\zeta)\right| \leq\left|f_{1}(\zeta)\right|+\left(M_{\alpha}+1\right) \epsilon_{1}|E(\zeta)|
$$

Lemma 6 implies, for $\zeta \in \overline{\mathbb{D}}\left(0, r_{0}\right),\left|f_{1}(\zeta)\right| \leq|\phi(\zeta)|+\epsilon_{1}$, so

$$
\left|f_{2}\right|(\zeta)<1-\left(M_{\alpha}+2\right) \epsilon_{1}+\epsilon_{1}+\left(M_{\alpha}+1\right) \epsilon_{1}=1,
$$

by our previous choice of $\epsilon_{1}$; and for $\zeta \in\left[r_{0}, 1\right),\left|f_{1}(\zeta)\right| \leq|F(\zeta)|+$ $\epsilon_{1}|E(\zeta)|$, so

$$
\left|f_{2}\right|(\zeta) \leq|F(\zeta)|+\epsilon_{1}|E(\zeta)|+\left(M_{\alpha}+1\right) \epsilon_{1}|E(\zeta)|<1,
$$


by our choice of $F$.

Let $\Omega=\left\{\zeta \in \mathbb{D}\right.$ st $\left.\left|f_{2}\right|(\zeta)<1\right\}$ : by the above, $\Omega$ is a neighborhood of $\overline{\mathbb{D}}\left(0, r_{0}\right) \cup\left[r_{0}, 1\right)$, which is simply connected by the maximum modulus principle. Let $\lambda$ be a Riemann mapping from $\mathbb{D}$ to $\Omega$, and set $\beta:=$ $\lambda^{-1}(\alpha)$. The desired map will be $\psi:=f_{2} \circ \lambda$. Since, for any $j, k \in$ $\{1, \ldots, N\}$,

$$
\begin{aligned}
d_{G}^{\mathbb{D}}\left(\lambda^{-1}\left(\alpha_{j}\right), \lambda^{-1}\left(\alpha_{k}\right)\right) & =d_{G}^{\Omega}\left(\alpha_{j}, \alpha_{k}\right) \leq d_{G}^{\mathbb{D}\left(0, r_{0}\right)}\left(\alpha_{j}, \alpha_{k}\right) \\
& =d_{G}^{\mathbb{D}}\left(\alpha_{j} / r_{0}, \alpha_{k} / r_{0}\right),
\end{aligned}
$$

we have

$$
\begin{aligned}
\delta(\beta) & \leq \delta\left(\left\{\lambda^{-1}\left(\alpha_{j}\right), 1 \leq j \leq N\right\}\right) \\
& \leq \delta\left(\left\{\alpha_{j} / r_{0}, 1 \leq j \leq N\right\}\right)<\delta_{1}(\alpha)+\epsilon .
\end{aligned}
$$

\section{References}

[A-T] E. Amar And P. Thomas, A notion of extremal analytic discs related to interpolation in the ball, Math. Ann. 300 (1994), 419-433.

[C] L. Carleson, An interpolation problem for bounded analytic functions, Amer. J. Math. 80 (1958), 921-930.

[Ga] J. B. Garnett, "Bounded Analytic Functions," Academic Press, 1981.

[Gl] J. Globevnik, Discs in the ball containing given discrete sets, Math. Ann. 281 (1988), 87-96.

[Ka] W. Kaplan, Approximation by entire functions, Mich. Math. J. 3 (1955), 43-52.

[Ru] W. Rudin, "Function Theory in the Unit Ball of $C^{n}$," Springer Verlag, 1980.

U.F.R. Mathematiques Informatiques et Gestion

Université Paul Sabatier

118 route de Narbonne

31062 Toulouse cedex FRANCE

Primera versió rebuda el 15 de Març de 1995,

darrera versió rebuda el 17 de Maig de 1995 